\title{
A new partnership for an impactful future
}

Starting from this issue (January, 2016), SCIENCE CHINA Life Sciences (SCLS) and The Chinese Biological Investigators Society (CBIS) will embark on a partnership with the goal of making SCLS a highly impactful journal in advancing research in life sciences.

SCLS is a member of the SCIENCE CHINA (formerly SCIENCE in CHINA) series of academic journals. The series were first published in 1950 to accompany the founding of the Chinese Academy of Sciences (CAS) and have since become leading academic journals in China. SCLS is currently co-sponsored by CAS and the National Natural Science Foundation of China (NNSFC) and co-published by Science in China Press and Springer Verlag Press. Since 2009, SCLS has been undertaking a reform effort to internationalize and enhance its scientific impact.

CBIS, formerly known as Ray Wu Society, is a not-forprofit professional organization of life sciences and primarily comprises scientists who were born in China and went on to obtain their Ph.D. and/or pursue postdoctoral training in North America, Europe and other foreign countries starting from the early 1980s. Its members hold professorships, including assistant, associate and full professors or the equivalents, in academic institutions as well as group leader or top management positions in industry. The society was formed in 1998 to honor Dr. Ray Wu for his outstanding scientific contributions in Biochemistry and Plant Biotechnology and, more importantly, for his unmatched leadership in initiating and organizing CUSBEA (ㄷhina- $\underline{\text { United }} \underline{\text { States }}$ $\underline{B}$ iochemistry and Molecular Biology Examination and $\underline{\text { Ad- }}$ ministration). This program, operated between 1982 and 1989, enabled about 400 gifted college students from mainland China to study for Ph.D. in life sciences in U.S. universities. Many of the trainees have since made significant scientific contributions in many fields of life sciences. The society changed to its current name in 2005, with blessing from $\mathrm{Dr}$. Wu, to reflect its expanding membership.

We are optimistic that the SCLS-CBIS partnership will be a successful one. As mentioned above, SCLS is co-sponsored by CAS and NNSFC, which are, respectively, the premier research institute and the main funding agency for basic research in China. This unique co-sponsorship guarantees that advancing scientific research is the only goal of SCLS. This partnership will now also make the journal CBIS's own, and we are confident that CBIS members will enthusiastically embrace this collaboration as it is a wonderful reflection of its history. There is a strong desire among CBIS members to follow Dr. Wu's example in contributing to scientific development in China and in promoting scientific exchange between China and the outside world. A previous collaboration in the 2000s between CBIS and NNSFC to improve the grant reviewing process at the foundation - under the leadership of Prof. Zuoyan Zhu, who was a deputy director of NNSFC at the time and is currently the editor general of SCIENCE CHINA-has turned out to be extremely successful.

Moreover, as a journal striving to become more impactful, we are willing to experiment using this partnership. Our focus will be on long-term impact on life science research rather than just short-term gains in impact factor. To achieve our goal, a North America Office will be established to enhance communication between the journal and

Citation: Zhong, W., Wang, D.C. (2016). A new partnership for an impactful future. Sci China Life Sci 59, 1-2. doi: 10.1007/s11427-016-5014-9 
scientists outside of China. A major effort initially will be to engage Chinese as well as non-Chinese scientists, particularly those at early stages of their career, in identifying areas

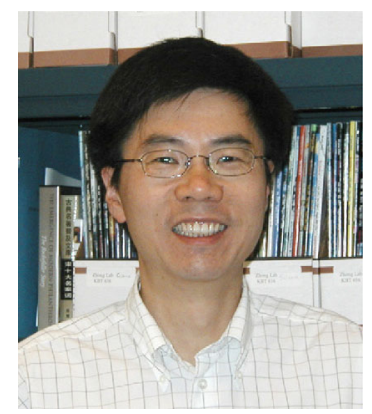

Weimin Zhong

President, The Chinese Biological Investigators Society Department of Molecular, Cellular and Developmental Biology Yale University, USA that perhaps are not trendy at the moment but may highly impact how life sciences advance. This is a challenging endeavor for this new partnership, but we are ready for it.

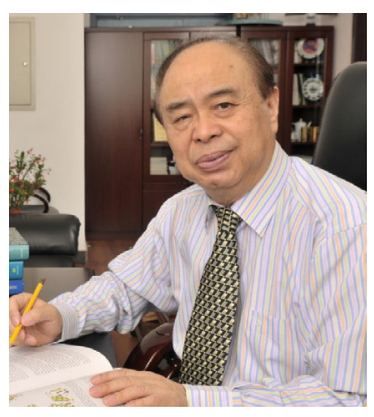

Da-Cheng Wang Editor-in-Chief, SCIENCE CHINA Life Sciences Institute of Biophysics Chinese Academy of Sciences, China

Open Access This article is distributed under the terms of the Creative Commons Attribution License which permits any use, distribution, and reproduction in any medium, provided the original author(s) and source are credited. 